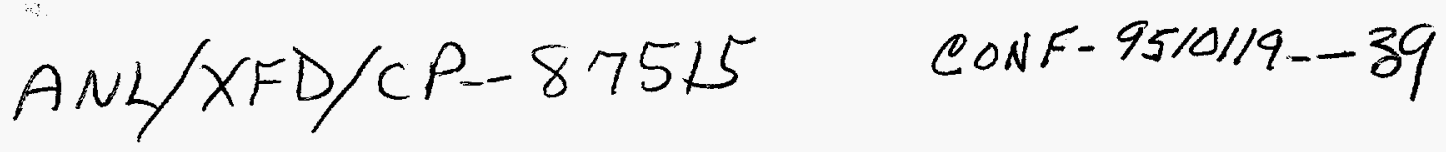

THE APS OPTICS TOPOGRAPHY STATION*

\author{
Szczesny (Felix) Krasnicki \\ Experimental Facilities Division, Advanced Photon Source, \\ Argonne National Laboratory, Argonne, IL 60439
}

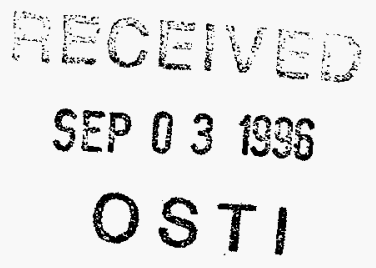

January 1996

The submitted manuscript has been authored
by a contractor of the U.S. Government
under contract No. W-31.109-ENG-38.
Accordingly, the U.S. Government retains a
nonexclusive, royalty-tree license to publish
or reproduce the published form of this
contribulion, or altow others to do so, for
U.S. Government purposes.

Presented at the SRI '95 APS X-ray Centennial Symposium/Seventh Users Meeting for the APS, Argonne, IL, October 16-20, 1995; to be published in the proceedings as a peer-reviewed volume of the Review of Scientific Instruments in CD-ROM format.

*This work supported by the U.S. Department of Energy, Basic Energy Sciences-Materials Sciences, under contract \#W-31-109-ENG-38. 


\section{DISCLAIMER}

This report was prepared as an account of work sponsored by an agency of the United States Government. Neither the United States Government nor any agency thereof, nor any of their employees, makes any warranty, express or implied, or assumes any legal liability or responsibility for the accuracy, completeness, or usefulness of any information, apparatus, product, or process disclosed, or represents that its use would not infringe privately owned rights. Reference herein to any specific commercial product, process, or service by trade name, trademark, manufacturer, or otherwise does not necessarily constitute or imply its endorsement, recommendation, or favoring by the United States Government or any agency thereof. The views and opinions of authors expressed herein do not necessarily state or reflect those of the United States Government or any agency thereof. 


\section{DISCLAIMER}

Portions of this document may be illegible in electronic image products. Images are produced from the best available original document. 


\title{
The APS optics topography station
}

\author{
Szczesny (Felix) Krasnicki \\ Experimental Facilities Division, Advanced Photon Source, Argonne National Laboratory, Argonne; \\ Illinois 60439
}

(Presented on 19 OCT 1995)

\begin{abstract}
An in-house station for topographic testing of $x$-ray optical elements for the Advanced Photon Source experimental beamlines was set up by the Experimental Facilities Division of Argonne National Laboratory. A new double-crystal x-ray diffractometer was designed and built keeping in mind the need for testing large crystals possibly attached to cooling manifolds and lines. A short description of the new facility is given. The instrument performance fully satisfies imposed requirements, and the machine was successfully used for testing several silicon and diamond crystals.
\end{abstract}

\section{INTRODUCTION}

Effective use of high intensity and high brilliance $x$-ray beams available at the Advanced Photon Source presents a multitude of challenges for designers of monochromators and analyzers. The design of crystals and their holders is usually complicated. It is easy therefore to introduce undesired and unexpected strains into the crystal elements. The need for a nondestructive and high sensitivity method of crystal testing like the topographic method 1,2 is obvious.

Recognizing such a need, the Experimental Facilities Division of Argonne National Laboratory decided to set up an in-house station for topographic testing of $x$-ray optics elements for the Advanced Photon Source. The objective was to build a machine capable of testing crystals of front face size up to $300 \mathrm{~mm} \times 90 \mathrm{~mm}$ and, if necessary, crystals attached to cooling manifolds or even mounted in cryostats. Also taking topograms in a relatively short time was assumed.

\section{MAIN DESIGN FEATURES}

In order to satisfy, at reasonable expenses, the requirements stated above, the design proceeded along the following guide lines.

1. The monochromatic and highly collimated beam obtained by reflection from the first crystal has to have a footprint approximately $80 \mathrm{~mm} \times 90 \mathrm{~mm}$. This could be obtained by the use of highly asymmetric monochromator crystals and by moving the $x$-ray point source far away from the diffractometer.

2. Testing of long crystals is to be accomplished in several steps by translation of a long asymmetric monochromator along the direction normal to the primary (i.e., the one coming directly from the source) beam and performing measurements at some selected translation positions.

3. Intensities of $x$-ray beams reflected from monochromator and sample crystals are to be monitored by two independent detection setups based on the use of scintillation detectors. Detectors should be mounted on translation stages in order to follow movement in the laboratory frame (caused by monochromator translation) of reflected beams.

4. The new double-crystal diffractometer should allow change of sample table position in the broad range of monochromator two-theta angles covering several essential reflections (mainly silicon samples were considered). No automation of such movement is necessary. The possibility of changing the distance between the diffractometer axes 1 and 2 would be useful.

5. For each crystal (diffractometer axis), the following freedoms of regulation should be present: rotation about the vertical (theta) axis, tilt rotation about a horizontal axis (if possible, parallel to a given reciprocal vector), and translation normal to the tilt axis. The sample (axis 2) theta rotation will consist of two subunits, i.e., coarse and fine (subsecond) rotation systems. The sizes of all stages will permit loading them with large crystals, if necessary, fixed to heavy holders. 6. Mechanical systems of theta rotation for axes 1 and 2 are to be interchangeable (fine rotation of the first crystal could be used in some cases when precise rotation of the second crystal would be impractical).

7. To lower the cost and accelerate the construction, the use of as many commercially available elements as possible, e.g., rotation tables, translation stages, is to be considered.

8. Use of a rotating anode (point focus) $x$-ray source and fast imaging methods are to be implemented.

\section{STATION DESCRIPTION}

The general layout of the topography station (not to scale) is shown in Fig. 1. The x-ray source (18 kW Rigaku rotating anode, $\mathrm{Cu}$ or Mo targets, point focus horizontal beam port, take-off angle 6 degrees) and the new doublecrystal diffractometer are installed in separate radiation enclosures. The distance between the source and the diffractometer axis 1 is about $1940 \mathrm{~mm}$. The primary beam is transported in a vacuum tube that ends about $180 \mathrm{~mm}$ from axis 1 . The tube end flange also serves as a base plate for: 1. a system of vertical and horizontal slits used for regulation of horizontal divergence of the beam impinging on the first crystal and, depending on the vertical size of the sample, for 


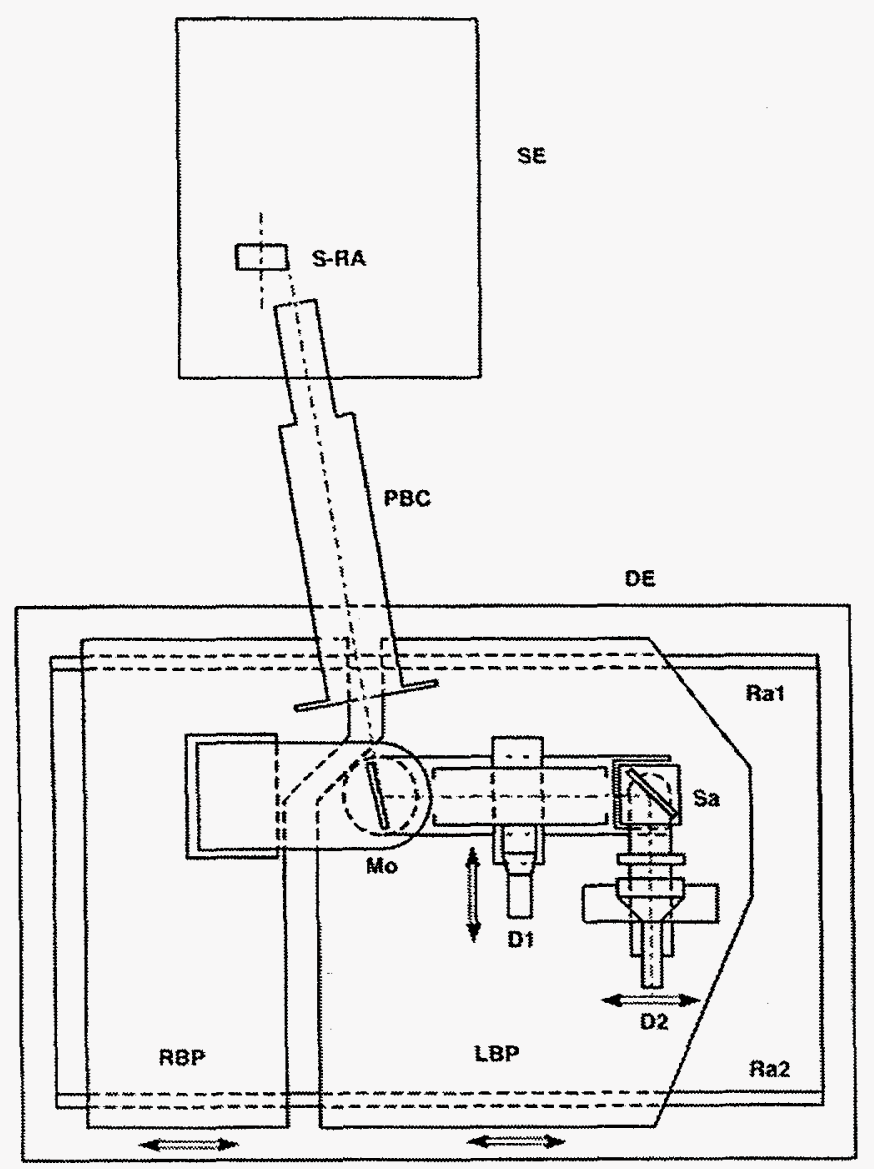

Fig. 1. General layout of the topography station. SE - source enclosure, S-RA - source-rotating anode $\mathrm{PBC}$ - primary beam collimator, DE - diffractometer enclosure Ra1 - rail 1, Ra2 - rail 2, Mo - monochromator, Sa - sample D1 - detector 1, D2 - detector 2,

RBP - right base plate, LBP - left base plate

vertical reduction of the primary beam entering the diffractometer enclosure,

2. additional lead enclosure surrounding the first crystal.

Positions of both vacuum tube ends can be adjusted in the vertical and horizontal directions allowing a search for the optimal position of the tube.

The diffractometer is mounted on a special base supported on four legs that are isolated from the lab floor by antivibrational pads. The base and the diffractometer have no direct contact with the radiation enclosure. The top part of the base consists of two separate horizontal big plates that can be moved independently along two common, exactly parallel, precise rails. Each plate is attached to the rails by means of four carriers (revolving, barrel linear bearings). The carriers, almost free of backlashes, and the long distances between them secure a high precision of plate translation. From the application point of view, the most critical error connected with the translation would be the possible minute rotation of plates about the vertical axis. Optical measurements showed that such rotation was within $+/-0.5$ arcsec on the travel distance of interest (approximately $+/-12 \mathrm{~mm}$ ).
The right (looking down the primary beam) base plate supports the mechanical system of axis 1 and a mechanism causing plate translation (this translation is motorized and automated). The left base plate supports the rest of the diffractometer, i.e., rotary stage 1 guiding the main arm, the detector 1 assembly, and the mechanical systems of axis 2 (theta rotation mechanisms, detector 2 subassembly, film cassette). Both parts of the diffractometer may be mechanically coupled forming a 'classical' double-crystal diffractometer, which as a whole may be translated (by the right plate mechanism) along the base rails.

The rotary stage 1 carries the main diffractometer arm, which couples the mechanical assemblies of axis 1 and axis 2 , and makes possible manual positioning of axis 2 at a desired two-theta angle(with respect to the primary beam) in the range 0 to 116 degrees. Arm construction in the form of a double slide enables changing of the distance between axis 1 and 2 in the range $560+/-64 \mathrm{~mm}$, with five discrete positions available. The main arm supports directly only the detector 1 assembly. It can be also translated along the arm. The detector 1 assembly consists of a translation stage (translation $+/-75 \mathrm{~mm}$ in the direction normal to the main arm axis) and a column carrying radiation shielding (rectangular tube $100 \mathrm{~mm} \times 100 \mathrm{~mm}$ also used for mounting collimating windows) with detector 1 attached to its side. Scintillation detector 1 registers photons scattered in the air (inside the shielding) between the monochromator and sample crystals.

The axis 2 assembly forms an independent single-axis diffractometer, which is coupled to the main arm by means of two vertical linear bearings. The assembly is supported directly on the left base plate by means of one central air pad and four support screws. Activation of the air pad lifts slightly the whole assembly and makes possible manual rotation of the rotary stage 1 and the main arm.

Both the axis 1 and axis 2 theta coarse-rotation mechanisms are almost identical. They use 6" dia., motorized (full revolution of the stepping motor corresponding to 2 degrees of theta change) rotation stages with encoders attached directly to tables along the rotation axes. For axis 2, the stage table carries an additional theta rotation subassembly for precise (fine) adjustment of the sample position. (If necessary, this fine rotation subassembly may be mounted on the axis 1 rotary table.) The fine rotation is achieved by pushing a long lever by a motorized leadscrew. Full revolution of the stepping motor corresponds to a 4-arcsec rotation of the sample. Use of a microstepping driver (at present, 50000 steps per revolution) provides very smooth crystal rotation with a motor resolution of 0.00008 arcsec. In practice, so far, steps not smaller than 0.1 arcsec were used for rocking curve measurements.

The top parts of both axis 1 and 2 assemblies are again almost identical and, in the present configuration, contain heavy $170 \mathrm{~mm} \times 170 \mathrm{~mm}$ tilt stages (the distance from the top surface of a stage to its axis of rotation is $220 \mathrm{~mm}$ ) and translation stages (travel $+/-25 \mathrm{~mm}$ in the direction normal to the tilt axis). 
As detector 2, a Bicron scintillation detector with a $5 "$ dia. $\mathrm{x} \mathrm{.063"} \mathrm{NaI}(\mathrm{Tl})$ crystal and a $0.002 "$ thick aluminum window was chosen. This size of the detector is sufficient to accept diffracted photons from about 4" dia. samples at theta Bragg close to 45 degrees. A system of lead windows and aluminum attenuators is used in front of the detector to adjust the collection area to the dimensions of a given sample and, if necessary, to reduce the total measured intensity. The detector is mounted on the top of a column fixed to the translation stage. It can therefore be translated $+1-75 \mathrm{~mm}$ in the direction normal to the beam diffracted from the sample.

A special platform supported by the axis 2 assembly or by a separate construction fixed to the base frame makes possible insertion of a film cassette between the sample and detector 2. For some applications, imaging by means of photographic material could be replaced by an electronic method, e.g., by CCD camera imaging. We are currently working on such a project.

A photograph showing the essential parts of the diffractometer is demonstrated in Fig. 2.

Some operations of the instrument are motorized and automated. At the present time, ten movements can be performed with the use of stepping motors. Four of them are supplied from microstepping drivers. Movements of the motors, the operation of rocking curve measurement, and printing and plotting are controlled by networked SUN stations and EPICS and IDL software available to all APS users.

\section{CONCLUDING REMARKS}

Since completion of the assembly works in September 1994 and calibration of the instrument with the x-ray beam, the new topographic station has been used extensively for testing high-heat-load silicon monochromators 3 manufactured by the APS Experimental Facilities Division and some diamond crystals. So far, all the work was

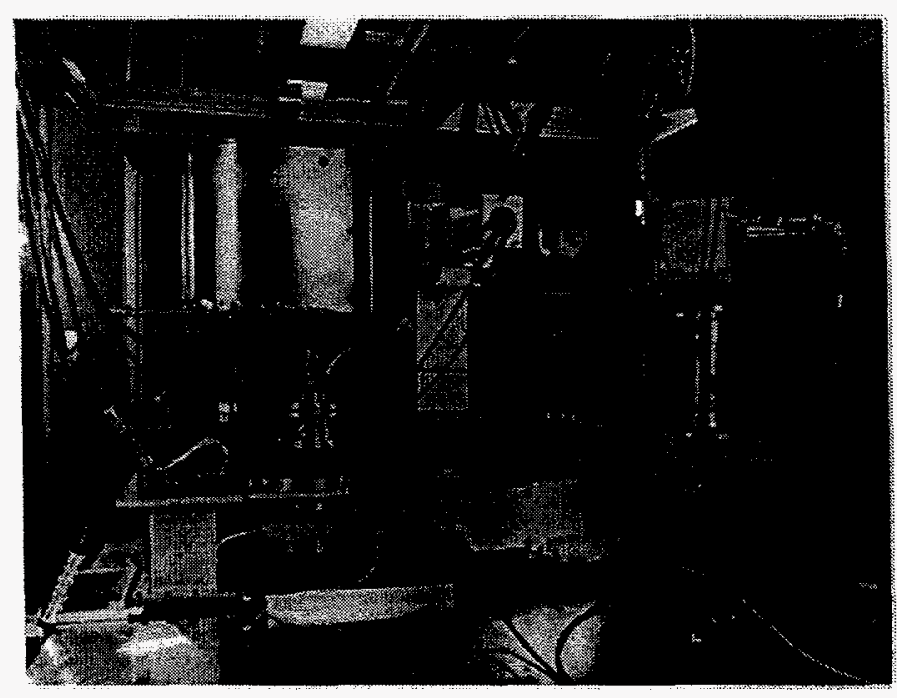

Fig. 2. Back view of the diffractometer. performed using $\mathrm{x}$-rays emerging from a $\mathrm{Cu}$ target (wavelength $1.54 \mathrm{~A}$ ). A highly asymmetric (asymmetry angle 46.7 degrees) silicon (333) monochromator was predominantly used. The monochromatic, extremely collimated $\mathrm{x}$-ray beam obtained from this monochromator usually covered most of investigated sample faces. Whenever dealing with unstrained samples, measurements yielded good quality topograms and rocking curves. Rocking curves were smooth and of correct widths thereby confirming proper operation of the instrument.

\section{ACKNOWLEDGMENTS}

The author would like to thank Ron Hopf, Kurt Goetze and Rod Salazar for their assistance in assembling and putting into operation the station, and Dr. Tim Mooney for his help in software development. This work was supported by the U.S. Department of Energy, BES-Materials Sciences, under contract No. W-31-109-ENG-38.

${ }^{1}$ B.K.Tanner, X-ray Diffraction Topography (Pergamon Press, Oxford, 1976).

${ }^{2}$ B.K.Tanner, D.K.Bowen, Characterization of Crystal Growth Defects by X-ray Methods (Plenum Press, New York and London, 1980).

${ }^{3}$ P. Fernandez, ANL, unpublished information, 1995. 